\title{
Spatial separation of state- and size-selected neutral clusters
}

\author{
Sebastian Trippel, ${ }^{1}$ Yuan-Pin Chang, ${ }^{1}$ Stephan Stern, ${ }^{1,2}$ Terry Mullins, ${ }^{1}$ Lotte Holmegaard, ${ }^{1}$ and Jochen Küpper ${ }^{1,2, *}$ \\ ${ }^{1}$ Center for Free-Electron Laser Science, DESY, Notkestrasse 85, 22607 Hamburg, Germany \\ ${ }^{2}$ University of Hamburg, Luruper Chaussee 149, 22761 Hamburg, Germany
}

(Received 20 December 2011; revised manuscript received 1 August 2012; published 10 September 2012)

\begin{abstract}
We demonstrate the spatial separation of the prototypical indole $\left(\mathrm{H}_{2} \mathrm{O}\right)$ clusters from the various species present in the supersonic expansion of mixtures of indole and water. The major molecular constituents of the resulting molecular beam are $\mathrm{H}_{2} \mathrm{O}$, indole, indole $\left(\mathrm{H}_{2} \mathrm{O}\right)$, and indole $\left(\mathrm{H}_{2} \mathrm{O}\right)_{2}$. It is a priori not clear whether such floppy systems are amenable to strong manipulation using electric fields. Here, we have exploited the cold supersonic molecular beam and the electrostatic deflector to separate indole $\left(\mathrm{H}_{2} \mathrm{O}\right)$ from the other molecular species as well as the helium seed gas. The experimental results are quantitatively explained by trajectory simulations, which also demonstrate that the quantum-state selectivity of the process leads to samples of indole $\left(\mathrm{H}_{2} \mathrm{O}\right)$ in low-lying rotational states. The prepared clean samples of indole $\left(\mathrm{H}_{2} \mathrm{O}\right)$ are ideally suited for investigations of the stereodynamics of this complex system, including time-resolved half-collision and diffraction experiments of fixed-in-space clusters. Our findings clearly demonstrate that the hydrogen bonded indole $\left(\mathrm{H}_{2} \mathrm{O}\right)$ complex behaves as a rigid molecule under our experimental conditions and that it can be strongly deflected.
\end{abstract}

DOI: 10.1103/PhysRevA.86.033202

PACS number(s): 36.40.-c, 37.20.+j, 06.60.Ei, 33.15.-e

The investigation of atomic and molecular aggregates-socalled clusters-provides detailed information for bridging the knowledge gap between microscopic atomic and molecular systems and macroscopic samples. This includes, for example, the observation of ferroelectricity and superconductivity [1], the investigation of proton wires [2], and the evidence for single water molecules acting as catalysts of chemical reactions [3]. Acid dissolution has been studied at the microscopic level by stepwise attachment of solvents to molecules one by one [4], and hydration shells are found to serve as heat sinks and to enhance the photostability of DNA [5]. Water clusters can have a significant effect on the chemistry and the climate in earth's atmosphere [6].

The actual dynamics of these processes could be investigated, for instance, using ultrashort-pulse electron $[7,8]$ or x-ray diffraction [9], diffraction-from-within [10], or high-harmonic-generation experiments [11]. However, these experiments rely on clean, homogeneous samples in order to produce so-called "molecular movies" for individual cluster sizes and isomers. Therefore, it is highly desirable to create pure samples of individual cluster species.

For charged particles, size selection can be performed according to the mass-to-charge ratio of the clusters. Large ionic systems can also be separated according to their shape in ion-mobility spectrometers [12]. The full separation of structural isomers (conformers) of specific sizes has so far been elusive, though. The preparation of state- and conformerselected samples of (small) neutral molecules has a long history going back to the seminal contributions by Stern and Gerlach and by Rabi [13-15]. For small molecules, static electric fields have extensively been used to select individual quantum states [16]. More recently, switched electric and magnetic fields have been employed in the selection and slowing of individual or small sets of eigenstates of small molecules [17-20]. For large molecules, dynamic (strong)

*jochen.kuepper@cfel.de; http://desy.cfel.de/cid/cmi focusing has to be employed $[21,22]$. The rotational state selectivity of this technique has been demonstrated [23,24] and it has been used for the spatial separation of individual conformers [25]. The state [26] and conformer [27] selection of large molecules has also been demonstrated using static inhomogeneous electric fields, going back to an original proposal for state selection by Stern [28].

It is a priori not clear, whether these techniques can be extended to complex molecules and clusters with large numbers of internal degrees of freedom, many of which have low excitation energies and will be excited even at low temperatures. Due to their large size (and correspondingly large moments of inertia) and their floppiness these systems have very high densities of states and, correspondingly, complex Stark energy curves with many real and avoided crossings. Indeed, it was concluded before that the "chaotic rotation" of such complex asymmetric rotors makes their manipulation very difficult [29]. Here, we set out to demonstrate that the electric deflection method is capable of spatially separating neutral polar molecules and clusters according to their size and conformation, providing unprecedented possibilities to investigate the structures and dynamics, even of complex aggregate systems.

We have chosen to demonstrate the spatial separation of the prototypical indole $\left(\mathrm{H}_{2} \mathrm{O}\right)$ cluster from the various species in a supersonic cluster expansion of indole $\left(\mathrm{C}_{8} \mathrm{H}_{7} \mathrm{~N}\right)$ and water $\left(\mathrm{H}_{2} \mathrm{O}\right)$ seeded in helium. Indole is the chromophore of the essential amino acid tryptophan and its intrinsic properties have been widely studied. Its emission properties are regularly used in fluorescence studies of proteins, where spectral shifts are directly related to the chromophores' environment [30]. The influence of water solvation on the indole chromophore has been discussed extensively, since it has strong influence on the electronic states, including the lowest-energy electronically excited states, which are considered to interchange their energetic order with the addition of water and other polar molecules [31]. The structure of the indole $\left(\mathrm{H}_{2} \mathrm{O}\right)$ complex was determined in the ground and electronically excited states [32,33] and the influence of water on the lowest 


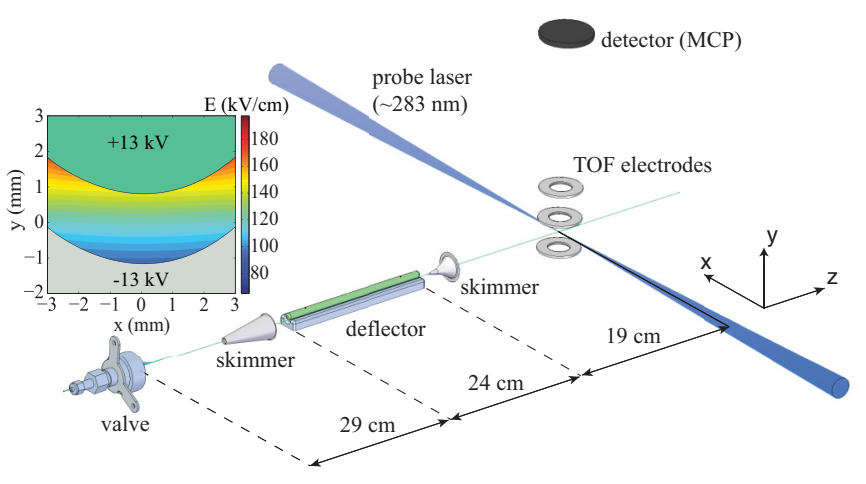

FIG. 1. (Color online) The experimental setup consists of a pulsed molecular beam source, a deflector, a time-of-flight mass spectrometer, and a pulsed dye laser. The inset shows a cut through the deflector and a contour plot of the electric field strength for an applied voltage of $26 \mathrm{kV}$.

electronic states discussed [34-36]. Indole $\left(\mathrm{H}_{2} \mathrm{O}\right)_{2}$ clusters were also investigated using sophisticated multiple-resonance spectroscopic techniques that showed, for instance, water-to$\pi$-orbital binding motifs [37]. The absorption spectra of indole in water solutions were interpreted using strong shifts and reordering of the molecules' electronic states [31].

Here, we experimentally demonstrate the preparation of a pure sample of the indole $\left(\mathrm{H}_{2} \mathrm{O}\right)$ clusters using the electric deflector to separate this species from the cluster expansion soup. We show simulations that reproduce the obtained results and will allow predictions for the preparation of further singlespecies samples even for such complex quantum systems.

The experimental setup is shown in Fig. 1. A supersonic expansion of a few mbar of indole and a trace of water molecules seeded in 50 bar of helium is provided by a pulsed Even-Lavie valve [38]. The valve is operated at a temperature of $40^{\circ} \mathrm{C}$ and at a repetition rate of $20 \mathrm{~Hz}$. Due to three-body collisions in the early phase of the expansion indole $\left(\mathrm{H}_{2} \mathrm{O}\right)_{n}$ clusters are formed. Under the employed conditions the indole $\left(\mathrm{H}_{2} \mathrm{O}\right)_{2}$ signal (density) is about an order of magnitude smaller than that of indole $\left(\mathrm{H}_{2} \mathrm{O}\right)$. Larger clusters are virtually absent from the beam. The resulting cluster beam passes a skimmer (Beam Dynamics model 50.8) with an orifice diameter of $2 \mathrm{~mm}$ placed $22 \mathrm{~cm}$ downstream from the nozzle and enters a differentially pumped vacuum chamber which houses the electric deflector. The length of the deflection device is $24 \mathrm{~cm}$ and the vertical distance between the two electrodes, a stainless steel trough and rod, is $2.3 \mathrm{~mm}$. We typically apply voltage differences of 0 to $26 \mathrm{kV}$ between the two electrodes. This results in electric field gradients as shown in Fig. 1, with field strengths and gradients of up to $120 \mathrm{kV} / \mathrm{cm}$ and $250 \mathrm{kV} / \mathrm{cm}^{2}$ in the center of the selector, respectively. The electric field gradient exerts a force on the molecules and clusters by which they are deflected towards the rod if they are in high-field-seeking states. Since the orifice diameter of the first skimmer and the distance of the two electrodes are comparable, we have moved this skimmer slightly towards the trough. This avoids the deflected beam crashing into the rod electrode and improves the sensitivity to deflected molecules. After passing a second skimmer with an orifice diameter of $2 \mathrm{~mm}$ mounted on a motorized translation stage the molecules enter the detection chamber. This chamber houses a time-of-flight (TOF) mass spectrometer. The position of the second skimmer is adjusted to maximize the transmission through the skimmer when the molecules are deflected. The molecular beam is crossed by a laser at $90^{\circ}$, in the center of the TOF-mass spectrometer. Indole and indole-water clusters are ionized via one-color resonance-enhanced multiphoton ionization (REMPI) at around $283 \mathrm{~nm}$ exploiting the lowest allowed electronic excitation of the indole chromophore. Due to the resonant excitation step involving different laser frequencies, the ionization is spectrally fully species selective. The laser light is provided by a nanosecond Nd:YAG laser (Innolas) pumped frequency doubled dye laser (Fine Adjustment). The light is focused by a 75-cm-focal-length lens and the typical pulse energy is a few $\mathrm{mJ}$. For detection, the resulting ions are accelerated towards a multichannel plate (MCP) detector and time-of-flight (TOF) mass spectra are recorded. Indole densities are determined from mass 117 (indole ${ }^{+}$) and indolewater densities are determined by summing masses 117 and 135 [indole $^{+}$and indole $\left(\mathrm{H}_{2} \mathrm{O}\right)^{+}$] following (neutral-speciesselective) resonance-enhanced ionization of the respective molecules or clusters. The focusing lens is mounted on a translation stage and spatial density profiles of the molecular beam are obtained by moving the lens perpendicular to the flight direction ( $y$ coordinate; see Fig. 1) of the molecular beam.

Figure 2 shows structures, molecular constants, and Stark energies of all relevant species. These are experimentally determined for $\mathrm{H}_{2} \mathrm{O}[39,40]$, indole $[36,41]$, and indole $\left(\mathrm{H}_{2} \mathrm{O}\right)$ $[33,36]$ and based on ab initio calculations (GAMESS-US [42]; B3LYP $\left./ 6-31+\mathrm{G}^{*}\right)$ for indole $\left(\mathrm{H}_{2} \mathrm{O}\right)_{2}$; the calculated structure and dipole moment are the same as the ones reported by Carney et al. $[43,44]$. The available experimental quartic centrifugal distortion constants for water, indole, and indole $\left(\mathrm{H}_{2} \mathrm{O}\right)$ are used in the calculations $[45,46]$. However, the resulting calculated deflection profiles using the rigid-rotor (not shown) and the semi-rigid-rotor [47] approximations are identical. The inset of Fig. 2(a) is a zoom into the Stark energies of water in
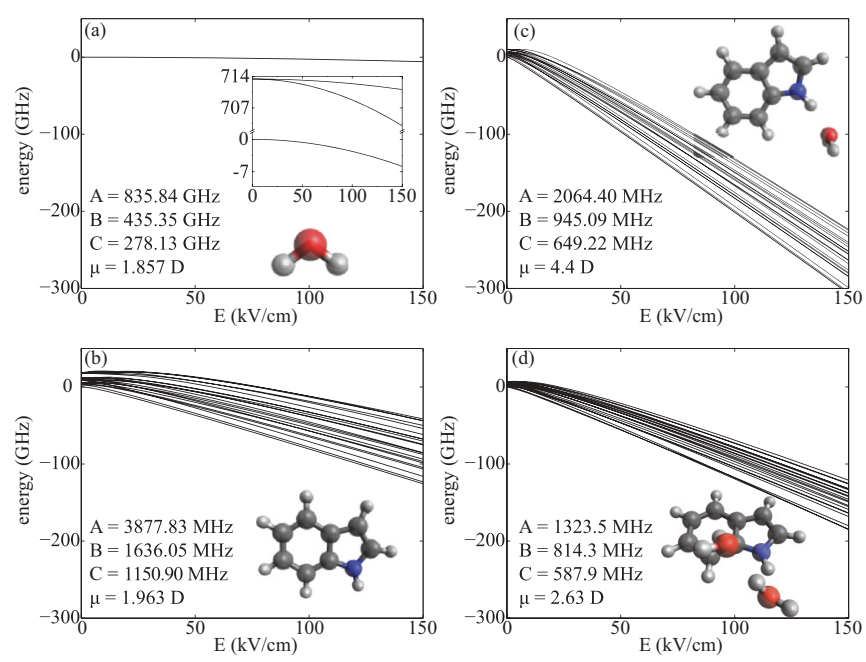

FIG. 2. (Color online) Structures, molecular constants, and Stark energies of (a) $\mathrm{H}_{2} \mathrm{O}$, (b) indole, (c) indole $\left(\mathrm{H}_{2} \mathrm{O}\right.$ ), and (d) indole $\left(\mathrm{H}_{2} \mathrm{O}\right)_{2}$; see text for details. 


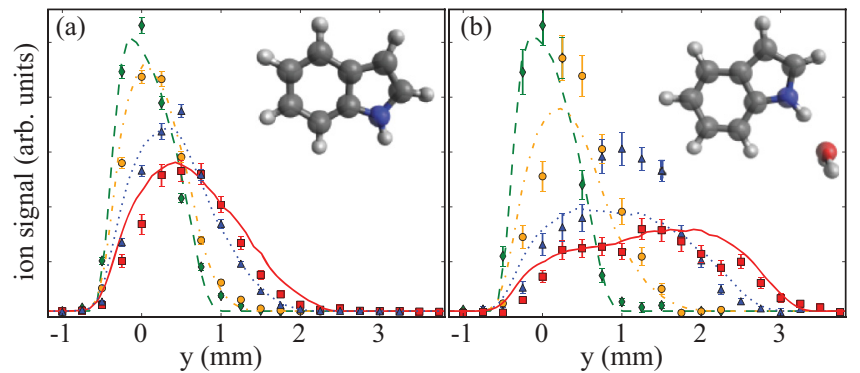

FIG. 3. (Color online) Deflection curves of (a) indole and (b) indole $\left(\mathrm{H}_{2} \mathrm{O}\right)$ for deflector voltages of 0 (green diamonds), $10 \mathrm{kV}$ (yellow circles), $20 \mathrm{kV}$ (blue triangles), and $26 \mathrm{kV}$ (red squares). The lines show the outcome of the respective trajectory calculations; see text for details.

order to visualize its small Stark shifts. All populated states of the molecules and clusters, at our experimental conditions, show a decrease of energy with increasing field strength for the relevant range of $\sim 50-150 \mathrm{kV} / \mathrm{cm}$. Thus, all molecules are in high-field seeking states. The change in energy in the strong electric field depends on the specific rotational quantum state. In general, the Stark effect decreases with increasing $J$ quantum number giving rise to the largest change in energy for the rotational ground states. Therefore, molecules in the lowest rotational states are deflected the most. Indole $\left(\mathrm{H}_{2} \mathrm{O}\right)$ shows the largest Stark effect of all molecules and clusters under consideration-its potential energy changes $50 \%$ more than for indole $\left(\mathrm{H}_{2} \mathrm{O}\right)_{2}$ and twice as much as for indole: If the clusters behave "well" (vide supra), the biggest change in the molecular beam profile, due to the interaction with the inhomogeneous electric field in the detector, clearly is expected for indole $\left(\mathrm{H}_{2} \mathrm{O}\right)$.

Figure 3 shows the indole and indole $\left(\mathrm{H}_{2} \mathrm{O}\right)$ density profiles of the molecular beam, obtained by scanning the focused REMPI laser beam perpendicular to the molecular beam direction, for deflector voltages of $0,10 \mathrm{kV}, 20 \mathrm{kV}$, and $26 \mathrm{kV}$. The width (full width at half maximum) of the undeflected beam is about $1 \mathrm{~mm}$, limited by the mechanical aperture between the first skimmer and the deflector trough in order to avoid collisions of the molecular beam with the rod and to increase the sensitivity to detect deflected molecules. The density profiles show that all molecules and clusters are deflected upwards and that they are dispersed as the deflector voltage is increased. This can be attributed to all quantum states being high-field seeking for electric field strengths present in the deflector and a variation (decrease) of the Stark effects with increasing rotational excitation, respectively; see Fig. 2. The lines in Fig. 3 correspond to simulated molecular beam profiles [46]. The force field inside the deflector has been derived from the calculated electric field and the Stark curves and the profiles have been obtained via Monte Carlo trajectory simulations of individual molecules. The good agreement between simulation and experimental data has been obtained by assuming a rotational temperature of $4 \mathrm{~K}$ for indole and of $6 \mathrm{~K}$ for indole $\left(\mathrm{H}_{2} \mathrm{O}\right)$ and by positioning the source and the two skimmers accordant to the experimental conditions.

The indole and water moieties of indole $\left(\mathrm{H}_{2} \mathrm{O}\right)$ are connected by a single hydrogen bond, and they can rotate with respect to one another about this single bond. This torsional

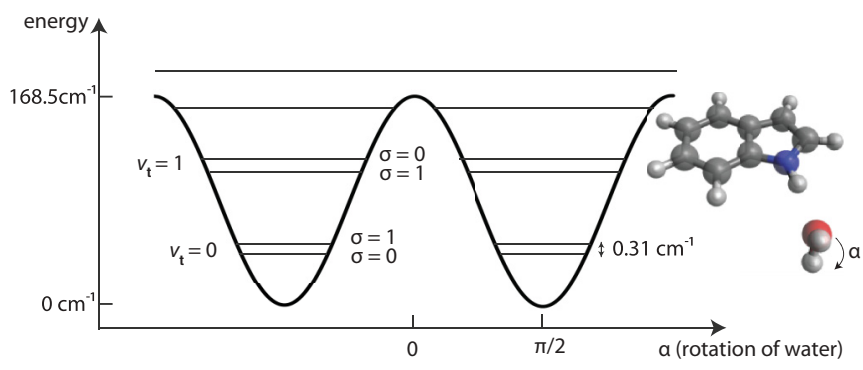

FIG. 4. (Color online) The torsion potential and energy level scheme of indole $\left(\mathrm{H}_{2} \mathrm{O}\right)$ in its vibronic ground state (excluding torsion).

motion introduces the complication that the cluster generally cannot be treated as a (semi)rigid rotor. However, under our experimental conditions the cluster regains rigidity due to the low temperature of our sample, the large rotational constant of the internal rotation of water, and the (dis)allowed symmetry couplings of the populated states: Consider the twofold symmetric potential energy curve of indole $\left(\mathrm{H}_{2} \mathrm{O}\right)$ shown in Fig. 4. The angle between the molecular plane of indole and the molecular plane of the water moiety is denoted by $\alpha$. The torsional energy levels are labeled by the torsional quantum number $v_{\mathrm{t}}$. Each torsional level is split due to tunneling splitting and the corresponding torsional sublevels are denoted by $\sigma=0,1$. The barrier height, $V_{2}=168.5 \mathrm{~cm}^{-1}$, the angle of the axis of rotation of the water moiety in the $b c$ symmetry plane of the cluster (corresponding to the $a b$ plane of bare indole) with respect to the $b$ axis, $\beta=55^{\circ}$, as well as the rotational constants have been taken from Ref. [32]. In order to determine the energy levels we have numerically solved the one-dimensional Schrödinger equation $[48,49]$. The included assumption that the rotating moiety is a symmetric top molecule is not strictly fulfilled for water. However, the principal axes of indole $\left(\mathrm{H}_{2} \mathrm{O}\right)$ do not change significantly when the water rotates and we can treat water as a symmetric top [32,50]. With this model, we calculate a subtorsional splitting in $v_{\mathrm{t}}=0$ of $0.30 \mathrm{~cm}^{-1}$ in agreement with the originally derived value of $0.31 \mathrm{~cm}^{-1}$ for this model [32]. In order to estimate the contribution of $v_{\mathrm{t}} \geqslant 1$ due to its initial state population prior to entering the strong field, we assume the population of the energy levels to follow a Boltzmann distribution. The energy difference between $\left|v_{\mathrm{t}}, \sigma\right\rangle=|0,0\rangle$ and $\left|v_{\mathrm{t}}, \sigma\right\rangle=|1,1\rangle$ is $70.9 \mathrm{~cm}^{-1}$ and the difference between $\left|v_{\mathrm{t}}, \sigma\right\rangle=|0,1\rangle$ and $\left|v_{\mathrm{t}}, \sigma\right\rangle=|1,0\rangle$ is $76.5 \mathrm{~cm}^{-1}$. This gives rise to a relative population of $v_{\mathrm{t}}=$ 1 with respect to $v_{\mathrm{t}}=0$ of less than $1 \%$ at vibrational temperatures at $20 \mathrm{~K}$ or below, as is expected for our supersonic expansion. Therefore, we neglect population with $v_{\mathrm{t}} \geqslant 1$ in our calculation, confirmed by the good agreement with the experimental results.

The two subtorsional states in $v_{\mathrm{t}}=0$ do not couple in the strong electric field of the deflector due to their symmetry (see Appendix A). Coupling to $v_{\mathrm{t}} \geqslant 1$ can be neglected, because the field in the deflector is not strong enough to efficiently couple $v_{\mathrm{t}}=0$ to these states (essentially because the energetic separation is too large). Therefore, under our well-controlled experimental conditions indole $\left(\mathrm{H}_{2} \mathrm{O}\right)$ can be treated as a (quasi)rigid molecule. 


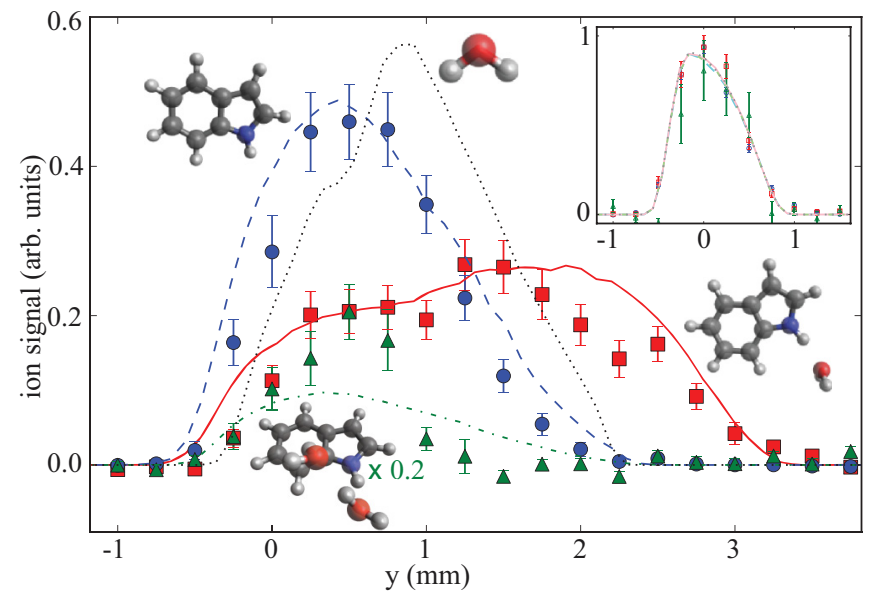

FIG. 5. (Color online) Normalized experimental (symbols) and simulated (lines) molecular beam profiles for water (dotted line), indole (blue circles-dashed line), indole $\left(\mathrm{H}_{2} \mathrm{O}\right)$ (red squares-solid line), and indole $\left(\mathrm{H}_{2} \mathrm{O}\right)_{2}$ (green triangles-dash-dotted line) at a deflector voltage of $26 \mathrm{kV}$. The inset shows the corresponding undeflected normalized beam profiles.

Figure 5 shows the experimental and simulated beam deflection profiles for indole, indole $\left(\mathrm{H}_{2} \mathrm{O}\right)$, and indole $\left(\mathrm{H}_{2} \mathrm{O}\right)_{2}$, and the simulated profiles for water, for voltages of 0 and $26 \mathrm{kV}$ applied across the deflector. The deflection-field-free density profiles are depicted in the inset of Fig. 5. All undeflected and deflected beam profiles have been normalized consistently to the field-free peak intensity for each species. This accounts for the unknown (relative) cross sections for ionizing the molecules and clusters and for the unknown number densities of the different species. The width of the undeflected beams is the same for all species. For strong electric fields applied, the beam profiles of all species broaden-due to the different effective dipole moments of the populated states-and shift upwards to larger $y$ values. Indole $\left(\mathrm{H}_{2} \mathrm{O}\right)$ is clearly more deflected than water, indole, and indole $\left(\mathrm{H}_{2} \mathrm{O}\right)_{2}$, as it is expected from the Stark curves shown in Fig. 2. We note that "exceptionally strong deflection" was reported for water in two of its $J=1$ states [51]. The reported behavior is reproduced by our simulations, but this level of deflection is not very strong on the scale of our experiment. These results (i.e., the profiles shown in Fig. 5) demonstrate that it is possible to perform experiments on pure samples of indole $\left(\mathrm{H}_{2} \mathrm{O}\right)$ by simply selecting the species present in the $y$ range $2.3-3.3 \mathrm{~mm}$. At $y=2.75 \mathrm{~mm}(3.0 \mathrm{~mm})$ the signal is $10 \%(4 \%)$ of the undeflected peak intensity with only the 290 (110) lowest rotational quantum states populated, instead of the 4600 states of indole $\left(\mathrm{H}_{2} \mathrm{O}\right)$ populated in the original molecular beam. Such state-selected samples have been shown to be very amenable to alignment and orientation control [26,52].

In conclusion, we have spatially separated indole $\left(\mathrm{H}_{2} \mathrm{O}\right)$ from a cluster beam containing water, indole, and various indole-water clusters as well as atomic helium. We have experimentally demonstrated that indole $\left(\mathrm{H}_{2} \mathrm{O}\right)$ is deflected more than the indole monomer and the indole $\left(\mathrm{H}_{2} \mathrm{O}\right)_{2}$ cluster. We have quantitatively simulated all experimental data using trajectory simulations. The good agreement of the simulations with the experiments demonstrate that the manipulation of complex molecular systems and even weakly bound clusters is possible and can be understood-if the initial temperature of the sample is low enough. These simulations also demonstrate that the indole $\left(\mathrm{H}_{2} \mathrm{O}\right)$ clusters are separated from $\mathrm{H}_{2} \mathrm{O}$ and, obviously, from the (undeflected) atomic helium seed gas as well as from larger water, indole, or indole-water clusters, whose contribution to the beam is minimal and which have smaller dipole-moment-to-mass ratios. Due to the state-specific deflection, it will also be possible to separate different structural isomers, which will occur for larger clusters [43] - analogous to the conformer separation of individual molecules [25,27].

The resulting pure samples of individual cluster species will allow for novel experiments investigating the structures and dynamics of these species. One particular experiment we foresee is the investigation of the energetics and stereodynamics, and their correlation, of half-collisions of indole $\left(\mathrm{H}_{2} \mathrm{O}\right)$ in the molecular frame. One would three-dimensionally orient [52] the molecules in the deflected pure sample, photodissociate the cluster species using various photon energies, and, successively, ionize the neutral water fragment from the reaction. The full momentum vector of the water molecule in the molecular frame would then be determined using ion imaging. Similar investigations using time-resolved gas-phase $\mathrm{x}$-ray and electron diffraction experiments are also envisioned [53]. Such experiments would provide novel details about the intermolecular interactions during, and their influence on, chemical reactions of complex molecules [54-56].

Note added in proof. Recently, in a related experiment the selective alternating-gradient focusing of benzonitrile and the benzonitrile-argon cluster was demonstrated [59].

\section{ACKNOWLEDGMENTS}

J.K. thanks David W. Pratt for the initiation of the research of indole and the indole-water complex. We thank L. Gumprecht, J. Schulz, K. Długołęcki, H. Mahn, and $H$. Chapman for help regarding the setup of the experiment, the DESY infrastructure groups for support, and HASYLAB/Petra III for hosting our laboratory. We acknowledge equipment loans from the Fritz Haber Institute, Berlin.

\section{APPENDIX: SYMMETRY PROPERTIES OF INDOLE $\left(\mathrm{H}_{2} \mathrm{O}\right)$ AND COUPLING BETWEEN SUBTORSIONAL LEVELS}

Here, we consider the symmetry properties of the torsional and subtorsional levels of indole $\left(\mathrm{H}_{2} \mathrm{O}\right)$ and the couplings between them due to an external dc electric field. The argumentation is equivalent to the case of phenol $\left(\mathrm{H}_{2} \mathrm{O}\right)$ [57]. Because it is important regarding the floppiness or rigidity of

TABLE I. Character tables for the molecular symmetry groups $\mathrm{G}_{4}$ and $\mathrm{G}_{2}[57,58]$.

\begin{tabular}{lrrrr|rrr}
\hline \hline $\mathrm{G}_{4}$ & $E$ & $(12)$ & $E^{*}$ & $(12)^{*}$ & $\mathrm{G}_{2}$ & $E$ & $(12)$ \\
\hline $\mathrm{A}_{1}$ & 1 & 1 & 1 & 1 & $\mathrm{~A}$ & 1 & 1 \\
$\mathrm{~A}_{2}$ & 1 & 1 & -1 & -1 & $\mathrm{~B}$ & 1 & -1 \\
$\mathrm{~B}_{1}$ & 1 & -1 & -1 & 1 & & & \\
$\mathrm{~B}_{2}$ & 1 & -1 & 1 & -1 & & & \\
\hline \hline
\end{tabular}


TABLE II. The symmetry classification of eigenstates of fieldfree indole $\left(\mathrm{H}_{2} \mathrm{O}\right)$ and the respective nuclear spin statistical weights (nssw).

\begin{tabular}{ccccccccccc}
\hline \hline$\sigma$ & $\mathrm{K}_{a}$ & $\mathrm{~K}_{c}$ & $\Gamma_{e}$ & $\Gamma_{v}$ & $\Gamma_{t}$ & $\Gamma_{r}$ & $\Gamma_{t} \otimes \Gamma_{r}$ & $\Gamma_{\mathrm{ns}}$ & $\Gamma_{\text {tot }}$ & $\mathrm{nssw}$ \\
\hline 0 & $e$ & $e$ & $\mathrm{~A}_{1}$ & $\mathrm{~A}_{1}$ & $\mathrm{~A}_{1}$ & $\mathrm{~A}_{1}$ & $\mathrm{~A}_{1}$ & $\mathrm{~B}_{2}$ & $\mathrm{~B}_{2}$ & 1 \\
0 & $o$ & $e$ & $\mathrm{~A}_{1}$ & $\mathrm{~A}_{1}$ & $\mathrm{~A}_{1}$ & $\mathrm{~A}_{1}$ & $\mathrm{~A}_{1}$ & $\mathrm{~B}_{2}$ & $\mathrm{~B}_{2}$ & 1 \\
0 & $e$ & $o$ & $\mathrm{~A}_{1}$ & $\mathrm{~A}_{1}$ & $\mathrm{~A}_{1}$ & $\mathrm{~A}_{2}$ & $\mathrm{~A}_{2}$ & $\mathrm{~B}_{2}$ & $\mathrm{~B}_{1}$ & 1 \\
0 & $o$ & $o$ & $\mathrm{~A}_{1}$ & $\mathrm{~A}_{1}$ & $\mathrm{~A}_{1}$ & $\mathrm{~A}_{2}$ & $\mathrm{~A}_{2}$ & $\mathrm{~B}_{2}$ & $\mathrm{~B}_{1}$ & 1 \\
1 & $e$ & $e$ & $\mathrm{~A}_{1}$ & $\mathrm{~A}_{1}$ & $\mathrm{~B}_{2}$ & $\mathrm{~A}_{1}$ & $\mathrm{~B}_{2}$ & $3 \mathrm{~A}_{1}$ & $\mathrm{~B}_{2}$ & 3 \\
1 & $o$ & $e$ & $\mathrm{~A}_{1}$ & $\mathrm{~A}_{1}$ & $\mathrm{~B}_{2}$ & $\mathrm{~A}_{1}$ & $\mathrm{~B}_{2}$ & $3 \mathrm{~A}_{1}$ & $\mathrm{~B}_{2}$ & 3 \\
1 & $e$ & $o$ & $\mathrm{~A}_{1}$ & $\mathrm{~A}_{1}$ & $\mathrm{~B}_{2}$ & $\mathrm{~A}_{2}$ & $\mathrm{~B}_{1}$ & $3 \mathrm{~A}_{1}$ & $\mathrm{~B}_{1}$ & 3 \\
1 & $o$ & $o$ & $\mathrm{~A}_{1}$ & $\mathrm{~A}_{1}$ & $\mathrm{~B}_{2}$ & $\mathrm{~A}_{2}$ & $\mathrm{~B}_{1}$ & $3 \mathrm{~A}_{1}$ & $\mathrm{~B}_{1}$ & 3 \\
\hline \hline
\end{tabular}

the indole $\left(\mathrm{H}_{2} \mathrm{O}\right)$ cluster in our experiment the derivation is provided here in detail.

We describe the internal motion of the water moiety with respect to the indole moiety using the principal axis method (PAM) [48,49]: We use the principal axes of the cluster as a coordinate system in combination with an internally rotating symmetric top moiety. The axis of internal rotation coincides with the symmetry axis of that symmetric top and, therefore, internal rotation does not change the moments of inertia of the overall rotation nor the cluster's principal axes. This condition is strictly speaking not fulfilled for the case of indole $\left(\mathrm{H}_{2} \mathrm{O}\right)$ since the rotating water moiety is an asymmetric top molecule. However, the moment of inertia of the rotating water molecule about its pseudosymmetry axis is small compared to the moment of inertia of the total molecule. The principal axes and moments of inertia of the cluster are only slightly and insignificantly altered by the rotation of the water molecule. The applicability of this approach is confirmed by related spectroscopic studies of hydrogen bound molecular clusters $[32,36,50,57]$.

In field-free space, indole $\left(\mathrm{H}_{2} \mathrm{O}\right)$ belongs to the molecular symmetry (MS) group $G_{4}=\left\{E,(12), E^{*},(12)^{*}\right\}$ [58] and the corresponding character table is provided in Table I. The symmetry of the total wave function $\Gamma_{\text {tot }}$ is of $B_{1}$ or $B_{2}$ symmetry since
TABLE III. The symmetry classification of eigenstates of indole $\left(\mathrm{H}_{2} \mathrm{O}\right)$ in a dc electric field and the respective nuclear spin statistical weights (nssw).

\begin{tabular}{ccccccccccc}
\hline \hline$\sigma$ & $\mathrm{K}_{a}$ & $\mathrm{~K}_{c}$ & $\Gamma_{e}$ & $\Gamma_{v}$ & $\Gamma_{t}$ & $\Gamma_{r}$ & $\Gamma_{t} \otimes \Gamma_{r}$ & $\Gamma_{\mathrm{ns}}$ & $\Gamma_{\mathrm{tot}}$ & $\mathrm{nssw}$ \\
\hline 0 & $e$ & $e$ & $\mathrm{~A}$ & $\mathrm{~A}$ & $\mathrm{~A}$ & $\mathrm{~A}$ & $\mathrm{~A}$ & $\mathrm{~B}$ & $\mathrm{~B}$ & 1 \\
0 & $o$ & $e$ & $\mathrm{~A}$ & $\mathrm{~A}$ & $\mathrm{~A}$ & $\mathrm{~A}$ & $\mathrm{~A}$ & $\mathrm{~B}$ & $\mathrm{~B}$ & 1 \\
0 & $e$ & $o$ & $\mathrm{~A}$ & $\mathrm{~A}$ & $\mathrm{~A}$ & $\mathrm{~A}$ & $\mathrm{~A}$ & $\mathrm{~B}$ & $\mathrm{~B}$ & 1 \\
0 & $o$ & $o$ & $\mathrm{~A}$ & $\mathrm{~A}$ & $\mathrm{~A}$ & $\mathrm{~A}$ & $\mathrm{~A}$ & $\mathrm{~B}$ & $\mathrm{~B}$ & 1 \\
1 & $e$ & $e$ & $\mathrm{~A}$ & $\mathrm{~A}$ & $\mathrm{~B}$ & $\mathrm{~A}$ & $\mathrm{~B}$ & $3 \mathrm{~A}$ & $\mathrm{~B}$ & 3 \\
1 & $o$ & $e$ & $\mathrm{~A}$ & $\mathrm{~A}$ & $\mathrm{~B}$ & $\mathrm{~A}$ & $\mathrm{~B}$ & $3 \mathrm{~A}$ & $\mathrm{~B}$ & 3 \\
1 & $e$ & $o$ & $\mathrm{~A}$ & $\mathrm{~A}$ & $\mathrm{~B}$ & $\mathrm{~A}$ & $\mathrm{~B}$ & $3 \mathrm{~A}$ & $\mathrm{~B}$ & 3 \\
1 & $o$ & $o$ & $\mathrm{~A}$ & $\mathrm{~A}$ & $\mathrm{~B}$ & $\mathrm{~A}$ & $\mathrm{~B}$ & $3 \mathrm{~A}$ & $\mathrm{~B}$ & 3 \\
\hline \hline
\end{tabular}

the total wave function must be antisymmetric with respect to the exchange of the two protons [operation (12)]. The symmetry of the electronic, vibrational, torsional, rotational, and nuclear spin states are summarized in Table II. In the last column, the resulting nuclear spin statistical weights (nssw) are given.

In an electric field the molecular symmetry group of indole $\left(\mathrm{H}_{2} \mathrm{O}\right)$ reduces to $G_{2}=\{E,(12)\}$, which contains only two irreducible representations, $A$ and $B$; the character table is given in Table I. The symmetries, under $G_{2}$, of all rotational quantum states of indole $\left(\mathrm{H}_{2} \mathrm{O}\right)$ in its vibronic ground state are obtained directly by correlating $A_{1,2}$ and $B_{1,2}$ symmetries in $G_{4}$ to $A$ and $B$ in $G_{2}$, respectively. They are provided in Table III. Torsional sublevels with the same quantum number $\sigma$ have the same symmetry ( $A$ for all $\sigma=0$ sublevels and $B$ for all $\sigma=1$ levels). The nonzero $\mu_{a}$ and $\mu_{b}$ components of the electric dipole moment are both of $A$ symmetry, because they do not change sign under the operation (12) [i.e., (12) does not change the dipole moment of the cluster]. Thus, an electric field mixes only states having the same $\sigma$. Consequently the subtorsional states within a given $v_{\mathrm{t}}$ do not mix. In our experiment more than $99 \%$ of the population resides in the $v_{\mathrm{t}}=0$ states (see earlier discussion of vibrational temperatures), and indole $\left(\mathrm{H}_{2} \mathrm{O}\right)$ can be treated as a rigid rotor molecule under our experimental conditions.
[1] R. Moro, X. Xu, S. Yin, and W. A. de Heer, Science 300, 1265 (2003).

[2] C. Tanner, C. Manca, and S. Leutwyler, Science 302, 1736 (2003).

[3] E. Voehringer-Martinez, B. Hansmann, H. Hernandez, J. S. Francisco, J. Troe, and B. Abel, Science 315, 497 (2007).

[4] S. Hurley, T. Dermota, D. Hydutsky, and A. Castleman, Science 298, 202 (2002).

[5] T. Elsaesser, Biol. Chem. 390, 1125 (2009).

[6] V. Vaida, J. Chem. Phys. 135, 020901 (2011).

[7] H. Ihee, V. Lobastov, U. Gomez, B. Goodson, R. Srinivasan, C. Ruan, and A. H. Zewail, Science 291, 458 (2001).

[8] B. J. Siwick, J. R. Dwyer, R. E. Jordan, and R. J. D. Miller, Science 302, 1382 (2003).
[9] H. N. Chapman, A. Barty, M. J. Bogan, S. Boutet, S. Frank, S. P. Hau-Riege, S. Marchesini, B. W. Woods, S. Bajt, W. H. Benner, R. A. London, E. Plönjes, M. Kuhlmann, R. Treusch, S. Düsterer, T. Tschentscher, J. R. Schneider, E. Spiller, T. Möller, C. Bostedt, M. Hoener, D. A. Shapiro, K. O. Hodgson, D. van der Spoel, F. Burmeister, M. Bergh, C. Caleman, G. Huldt, M. M. Seibert, F. R. N. C. Maia, R. W. Lee, A. Szöke, N. Timneanu, and J. Hajdu, Nature Phys. 2, 839 (2006).

[10] A. Landers, T. Weber, I. Ali, A. Cassimi, M. Hattass, O. Jagutzki, A. Nauert, T. Osipov, A. Staudte, M. H. Prior, H. Schmidt-Böcking, C. L. Cocke, and R. Dörner, Phys. Rev. Lett. 87, 013002 (2001).

[11] J. Itatani, J. Levesque, D. Zeidler, H. Niikura, H. Pépin, J. C. Kieffer, P. B. Corkum, and D. M. Villeneuve, Nature (London) 432, 867 (2004). 
[12] G. von Helden, T. Wyttenbach, and M. T. Bowers, Science 267, 1483 (1995).

[13] W. Gerlach and O. Stern, Z. Phys. 9, 349 (1922).

[14] I. I. Rabi, S. Millman, P. Kusch, and J. R. Zacharias, Phys. Rev. 55, 526 (1939).

[15] Incidentally, a lot of the relevant early work is based on Rabi's visit of, and collaboration with, the group of Otto Stern in Hamburg in the late 1920s.

[16] J. Reuss, in Atomic and Molecular Beam Methods, edited by G. Scoles (Oxford University Press, New York, 1988), Vol. I, Chap. 11, pp. 276-292.

[17] S. Y. T. van de Meerakker, H. L. Bethlem, and G. Meijer, Nature Phys. 4, 595 (2008).

[18] M. Schnell and G. Meijer, Angew. Chem. Int. Ed. 48, 6010 (2009).

[19] M. T. Bell and T. P. Softley, Mol. Phys. 107, 99 (2009).

[20] S. D. Hogan, M. Motsch, and F. Merkt, Phys. Chem. Chem. Phys. 13, 18705 (2011).

[21] D. Auerbach, E. E. A. Bromberg, and L. Wharton, J. Chem. Phys. 45, 2160 (1966).

[22] H. L. Bethlem, M. R. Tarbutt, J. Küpper, D. Carty, K. Wohlfart, E. A. Hinds, and G. Meijer, J. Phys. B 39, R263 (2006).

[23] K. Wohlfart, F. Grätz, F. Filsinger, H. Haak, G. Meijer, and J. Küpper, Phys. Rev. A 77, 031404(R) (2008).

[24] S. Putzke, F. Filsinger, H. Haak, J. Küpper, and G. Meijer, Phys. Chem. Chem. Phys. 13, 18962 (2011).

[25] F. Filsinger, U. Erlekam, G. von Helden, J. Küpper, and G. Meijer, Phys. Rev. Lett. 100, 133003 (2008).

[26] L. Holmegaard, J. H. Nielsen, I. Nevo, H. Stapelfeldt, F. Filsinger, J. Küpper, and G. Meijer, Phys. Rev. Lett. 102, 023001 (2009).

[27] F. Filsinger, J. Küpper, G. Meijer, J. L. Hansen, J. Maurer, J. H. Nielsen, L. Holmegaard, and H. Stapelfeldt, Angew. Chem. Int. Ed. 48, 6900 (2009).

[28] O. Stern, Z. Phys. 39, 751 (1926).

[29] M. Abd El Rahim, R. Antoine, M. Broyer, D. Rayane, and P. Dugourd, J. Phys. Chem. A 109, 8507 (2005).

[30] J. T. Vivian and P. R. Callis, Biophys. J. 80, 2093 (2001).

[31] H. Lami and N. Glasser, J. Chem. Phys. 84, 597 (1986).

[32] T. M. Korter, D. W. Pratt, and J. Küpper, J. Phys. Chem. A 102, 7211 (1998).

[33] S. Blanco, J. Lopez, J. Alonso, P. Ottaviani, and W. Caminati, J. Chem. Phys. 119, 880 (2003).

[34] C. Brand, J. Küpper, D. W. Pratt, W. L. Meerts, D. Krügler, J. Tatchen, and M. Schmitt, Phys. Chem. Chem. Phys. 12, 4968 (2010).

[35] J. Küpper, D. W. Pratt, L. Meerts, C. Brand, J. Tatchen, and M. Schmitt, Phys. Chem. Chem. Phys. 12, 4980 (2010).

[36] C. Kang, T. M. Korter, and D. W. Pratt, J. Chem. Phys. 122, 174301 (2005).

[37] J. R. Carney, F. C. Hagemeister, and T. S. Zwier, J. Chem. Phys. 108, 3379 (1998).
[38] U. Even, J. Jortner, D. Noy, N. Lavie, and N. Cossart-Magos, J. Chem. Phys. 112, 8068 (2000).

[39] F. C. De Lucia, P. Helminger, R. L. Cook, and W. Gordy, Phys. Rev. A 5, 487 (1972).

[40] S. L. Shostak, W. L. Ebenstein, and J. S. Muenter, J. Chem. Phys. 94, 5875 (1991).

[41] W. Caminati and S. Dibernardo, J. Mol. Struct. 240, 253 (1990).

[42] M. S. Gordon and M. W. Schmidt, in Theory and Applications of Computational Chemistry: The First Forty Years, edited by C. E. Dykstra, G. Frenking, K. S. Kim, and G. E. Scuseria (Elsevier, Amsterdam, 2005).

[43] J. R. Carney and T. S. Zwier, J. Phys. Chem. A 103, 9943 (1999).

[44] K. R. F. Somers, E. S. Kryachko, and A. Ceulemans, Chem. Phys. 301, 61 (2004).

[45] J. K. G. Watson, in Vibrational Spectra and Structure, edited by J. R. Durig (Marcel Dekker, New York, 1977), Vol. VI, Chap. 1.

[46] F. Filsinger, J. Küpper, G. Meijer, L. Holmegaard, J. H. Nielsen, I. Nevo, J. L. Hansen, and H. Stapelfeldt, J. Chem. Phys. 131, 064309 (2009).

[47] The "semirigid" refers to centrifugal distortion effects, which are known from high-resolution spectroscopic studies, but which are not necessary in our simulations; as discussed in the main text.

[48] J. D. Lewis, T. B. Malloy, T. H. Chao, and J. Laane, J. Mol. Struct. 12, 427 (1972).

[49] W. Gordy and R. L. Cook, Microwave Molecular Spectra, 3rd ed. (John Wiley \& Sons, New York, 1984).

[50] M. Gerhards, M. Schmitt, and K. Kleinermanns, J. Chem. Phys. 104, 967 (1996).

[51] R. Moro, J. Bulthuis, J. Heinrich, and V. V. Kresin, Phys. Rev. A 75, 013415 (2007).

[52] I. Nevo, L. Holmegaard, J. H. Nielsen, J. L. Hansen, H. Stapelfeldt, F. Filsinger, G. Meijer, and J. Küpper, Phys. Chem. Chem. Phys. 11, 9912 (2009).

[53] F. Filsinger, G. Meijer, H. Stapelfeldt, H. Chapman, and J. Küpper, Phys. Chem. Chem. Phys. 13, 2076 (2011).

[54] A. G. Suits, Acc. Chem. Res. 41, 873 (2008).

[55] D. Townsend, S. A. Lahankar, S. K. Lee, S. D. Chambreau, A. G. Suits, X. Zhang, J. Rheinecker, L. B. Harding, and J. M. Bowman, Science 306, 1158 (2004).

[56] J. Mikosch, S. Trippel, C. Eichhorn, R. Otto, U. Lourderaj, J. X. Zhang, W. L. Hase, M. Weidemüller, and R. Wester, Science 319, 183 (2008).

[57] G. Berden, W. L. Meerts, M. Schmitt, and K. Kleinermanns, J. Chem. Phys. 104, 972 (1996).

[58] P. R. Bunker and P. Jensen, Fundamentals of Molecular Symmetry, Series in Chemical Physics (Institute of Physics Publishing, Bristol, UK, 2005).

[59] S. Putzke, F. Filsinger, J. Küpper, and G. Meijer, arXiv:1208.3188 [J. Chem. Phys. (2012) (in press)]. 